BMJ Open Diabetes Research \& Care

\title{
Characteristics and morphology of lipohypertrophic lesions in adults with type 1 diabetes with ultrasound screening: an exploratory observational study
}

\author{
Rabab Hashem, ${ }^{1}$ Henrietta Mulnier, ${ }^{1}$ Haya Abu Ghazaleh, ${ }^{1}$ Susan Halson-Brown, ${ }^{2}$ \\ Maria Duaso, ${ }^{1}$ Rebecca Rogers, ${ }^{1}$ Janaka Karalliedde (D) , ${ }^{3}$ Angus Forbes (D) ${ }^{1}$
}

To cite: Hashem R, Mulnier H, Abu Ghazaleh $\mathrm{H}$, et al. Characteristics and morphology of lipohypertrophic lesions in adults with type 1 diabetes with ultrasound screening: an exploratory observational study. BMJ Open Diab Res Care 2021;9:e002553. doi:10.1136/ bmjdrc-2021-002553

\section{- Additional supplemental material is published online only. To view, please visit the journal online (http://dx.doi. org/10.1136/bmjdrc-2021- 002553)}

Received 17 August 2021 Accepted 14 November 2021

D Check for updates

\section{(c) Author(s) (or their} employer(s)) 2021. Re-use permitted under CC BY-NC. No commercial re-use. See rights and permissions. Published by BMJ.

${ }^{1}$ Division of Care in Long-term Conditions, King's College London, London, UK ${ }^{2}$ Department of Women \& Children's Health, King's College London, London, UK ${ }^{3}$ Department of Diabetes, King's College London, London, UK

\section{ABSTRACT}

Introduction Lipohypertrophy is a common complication of exposure to insulin therapy. Despite the prevalence of lipohypertrophy and its potentially hazardous effects on glucose regulation, it remains a relatively understudied problem in diabetes. The objective of this study was to characterize lipohypertrophic tissue using ultrasound in adults with type 1 diabetes.

Research design and methods An observational study of 74 people with type 1 diabetes from a diabetes center in South East London. Participants' insulin exposed areas were scanned with ultrasound, with a high-frequency linear probe (6-13 MHz). The observed tissue changes were described, measured and graded according to nodule size and thickness of the dermal layer.

Results Participants mean age and diabetes duration were $40.6( \pm 14.2)$ and $18.3( \pm 10.9)$ years, respectively, and $60 \%(n=44)$ were male. A total of 740 lipohypertrophic nodules were observed, ranging from $1.8 \mathrm{~mm}$ to $40 \mathrm{~mm}$ in width. The mean (SD/range) number of nodules per participants was $10.4( \pm 6.2 / 1-29)$. Delineation between the dermal layers was disrupted in all current injection sites. In 52 participants (70\%), there was a 30\% increase in dermal thickness compared with local none injected tissue, and in 36 participants (48\%) the increase was 50\%. The level of thickness was $>3 \mathrm{~mm}$ in the abdominal areas of $22(40 \%)$ of these participants and in thighs of eight participants (17.8\%). Hypoechogenic areas suggestive of necrotic tissue were observed within the lipohypertrophic nodules of $22(30 \%)$ participants. Needle length and nodule depth were correlated $(r=0.69, p<0.001)$. A conceptual model of the insulin exposed tissue changes observed was constructed.

Conclusions The study confirms that insulin-exposed tissue changes are heterogenous and has provided conceptual and grading frameworks for classifying these changes. Further studies are required to establish the clinical implications of these classifications, in relation to glucose regulation and other clinical parameters.

\section{BACKGROUND}

Lipohypertrophy is a common complication following exposure to insulin therapy.

\section{Significance of this study}

What is already known about this subject?

- Lipohypertrophy is a common problem associated with insulin exposure in people with diabetes. Lipohypertrophy can impede insulin absorption impacting on glucose control. There is currently a lack of theoretical, pathological or clinical models to frame how to detect, characterize and manage lipohypertrophy.

\section{What are the new findings?}

- The pattern of tissue change observed in relation to tissue exposure is more complex than just lipohypertrophy. In addition to lipohypertrophic nodules, there is evidence of wider tissue thickening and dermal disruption.

- The study has revealed some novel features associated with lipohypertrophy, identifying areas of reduced echogenicity that may indicate low blood flow and potential necrotic tissue.

- Lipohypertrophic nodules were concentrated in common injection areas (lower abdomen and thighs), and their depth was correlated with needle depth.

- The characteristics observed in the study have been used to construct a conceptual model detailing the different dermal and subcutaneous tissue changes associated with insulin exposure.

How might these results change the focus of research or clinical practice?

- This study provides a step toward the development of more clinically effective strategies for detecting insulin-related tissue changes; further work is required to test the clinical validity of the ultrasoundgrading and detection methods used in this study.

Lipohypertrophy is characterized by the enlargement (hypertrophy) and proliferation (hyperplasia) of adipocytes in the subcutaneous tissue arising from the anabolic effect of exogenous insulin exposure. ${ }^{1}$ The anabolic 
effect of insulin involves the activation of insulin growth factor receptors on the adipocyte cell membrane. ${ }^{2}$ It has also been suggested that insulin exposure and repeated needle penetration may increase the density of the subcutaneous tissue through an inflammatory response, with macrophages forming dense plaques of tissue. ${ }^{34}$ The prevalence of lipohypertrophy in the diabetes population has been varyingly reported, reflecting the different methods used for case definition and study populations. A recent meta-analysis of 45 studies reported a pooled prevalence estimate of $41.8 \%$ (95\% CI $35.9 \%$ to $47.6 \%$ ) in insulin users, ${ }^{5}$ confirming that lipohypertrophy is a common problem.

In terms of the clinical effect of lipohypertrophy, injections into lipohypertrophic tissue can attenuate insulin absorption and action. ${ }^{6-8}$ Lipohypertrophic tissue can be fibrous, resulting in reduced blood flow in the subcutaneous tissue, impeding insulin absorption. ${ }^{9}$ Glucose clamping studies have shown diminished insulin action in lipohypertrophic areas with corresponding elevations in glucose $;{ }^{6}$ in one study, a $20 \%-25 \%$ reduction in insulin effect was reported. ${ }^{10}$ Hence, lipohypertrophy may lead to inconsistent insulin action and glucose variability, with the additional hazard of hypoglycemia if insulin is injected into unaffected tissue. Studies have reported associations between lipohypertrophy and glucose variability ${ }^{11-13}$ and with suboptimal glycemic control. ${ }^{14-16}$ However, these were cross-sectional studies comparing people with and without lipohypertrophy, and unadjusted for the multiple extraneous factors that could explain these observations.

Optimal methods for the clinical assessment, classification and detection of lipohypertrophy remain elusive. While there are consensus guidelines for screening lipohypertrophy via palpation or visual assessment, ${ }^{17} 18$ they focus on detecting defined nodules rather than the general tissue disruption associated with lipohypertrophic areas. ${ }^{19}$ Hence, ultrasound is increasingly being considered as a method for detecting lipohypertrophy as it provides more detailed imaging of the subcutaneous space. Ultrasound has been shown to be around $50 \%$ more sensitive in detecting lipohypertrophy compared with palpation, revealing lipohypertrophic lesions and tissue changes that are not detectable with palpation. ${ }^{20}$ However, while ultrasound provides a more detailed visualization of lipohypertrophy, it is not clear whether the additional tissue changes observed are important, as there are no current recognized thresholds for clinically significant lipohypertrophy.

Despite the prevalence of lipohypertrophy and its potentially hazardous effects on glucose regulation, it remains a relatively understudied problem in diabetes. While the hazards of lipodystrophy have been documented since the inception of exogenous insulin therapy, we do not have robust theoretical, pathological or clinical models to frame how we detect, characterize and manage lipohypertrophy. In this paper, we present some novel insights into the characteristics of lipohypertrophic lesions following ultrasonic examination, along with the dermal and subcutaneous damage associated with insulin exposure in adults with type 1 diabetes.

\section{RESEARCH DESIGN AND METHODS}

We undertook a clinical observational study to characterize lipohypertrophic tissue in adults with type 1 diabetes, considering their morphology, clinical characteristics and anatomical distribution. The study was conducted in the diabetes' clinic of a large teaching hospital in South East London, UK.

\section{Study participants}

Adults with type 1 diabetes were recruited to the study following referral from their clinician with suspected lipohypertrophy. Participants were recruited based on the following eligibility criteria: type 1 diabetes and using insulin for $>3$ years; aged $>20$ years; treated with multiple daily insulin injections $\geq 4$ per day $(60 \%$ with five main injections and $40 \%$ with four main injections, the number of daily correction doses was not recorded); and using the same insulin type and delivery method for the last 6 months. We excluded people who: were using a Continous Subcutaenous Insulin Infusion (CSII) pump; had other conditions associated with lipodystrophies; or had serious mental or physical illness. Given the selective criteria of suspected lipohypertrophy, and the high background prevalence, we estimated that 60 participants would be adequate to identify the common characteristics of lipohypertrophy in this population (a power calculation was not performed as the study did not test any hypotheses and was purely descriptive in nature).

\section{Scanning procedures}

Each participant was scanned following a standard operating procedure (SOP), using a high caliber ultrasound machine (The Sonosite X-Porte) with a high-frequency linear probe $(6-13 \mathrm{MHz})$. The scanning was performed, and images were recorded by one diabetes specialist nurse trained in ultrasound scanning under the supervision of a senior sonographer. The thickness of the dermal and subcutaneous tissue layers was measured at each insulin injection site and a comparison set of measurements taken from local tissue unexposed to insulin at the same anatomical site as a normal reference for each injection site. Lipohypertrophic tissues were identified as areas of increased reflectivity - hyperechoic tissue, indicating greater tissue density. Where tissue had formed identifiable nodules, these were measured vertically and horizontally at the widest point for width. The depth of the center of each lipohypertrophic area was also measured to compare with current needle length. During scanning, any other abnormal changes to the tissue observed were labeled on the images for later interpretation by the research team. Control sites of non-insulin exposed tissue for comparison were identified following the SOP, which indicated reference points in the local anatomical regions being scanned. Participants were then asked to 
verify whether they ever injected into these reference areas, if they had injected there, then they identified the nearest non-insulin exposed area. Following scanning, participants were: given advice on the extent and locations of any lipohypertrophic areas and adviced to avoid these areas; adviced on insulin doses to reduce the risk of hypoglycemia when injecting in lipohypertrophy free sites; and then followed up clinically. An SOP of the scanning method is available in online supplemental appendix 1 .

\section{Characterization methods}

The ultrasound scans for each participant were examined to review and measure all the identified nodules and subcutaneous changes. To characterize lipohypertrophy, we developed ultrasound-grading criteria based on current literature and the findings of a preliminary study of ultrasound-examined lipohypertrophy. ${ }^{21}$ The grading incorporated two types of tissue change: lipohypertrophic nodules (areas of increased reflectivity with an apparent nodule structure) and diffuse tissue (areas of general increased reflectivity without a defined structure). When the edges of the nodule were not clearly seen, images were considered in both the longitudinal and transverse planes to confirm the nodule margins. ${ }^{22}$ The ultrasound-grading criteria were: grade 0 (normal - no evident nodules or diffuse areas); grade 1 (diffuse areas of the injected subcutaneous tissue without clear lipohypertrophic nodules); grade 2 (nodules $1-5.9 \mathrm{~mm}$ ); grade 3 (nodules $6-9.9 \mathrm{~mm}$ ); and grade 4 (nodules $\geq 10 \mathrm{~mm}$ ). The size parameter was the width of the nodule at the widest point. The lipohypertrophy patterns observed on the scans of each participant were described and graded with reference to the presentation type (diffuse and/or nodular formation); location and distribution; and the width of the lipohypertrophic nodules. These data were used to grade and map the lipohypertrophic regions. The anatomical locations of lipohypertrophic nodules were collated to map the distribution of the observed lipohypertrophy. To characterize the dermal observations, the percentage increase in dermal thickness was calculated using the site dermal thickness compared with the local non-injected site reference and graded as follows: grade 1: normal, with dermal thickness $<2 \mathrm{~mm}^{23}$; grade 2: $2-2.9 \mathrm{~mm}$; and grade $3: \geq 3 \mathrm{~mm}$. We also looked at the scanned images to identify any additional features of insulin exposure in the dermis or subcutaneous tissue or within the presenting nodules.

\section{Demographic and clinical data}

Participants' age, gender, body mass index, ethnicity and duration of diabetes were recorded. Clinical data included: glycated hemoglobin (HbA1c); needle length; estimated insulin requirement ( 0.6 units per $\mathrm{kg}$ or body weight); and insulin doses (total daily insulin, basal and bolus insulin doses).
Table 1 Demographic and clinical characteristics of participants

\begin{tabular}{ll}
\hline Patient characteristics & Total $\mathbf{n}=\mathbf{7 4}$ \\
\hline Mean age in years (SD) & $40.6 \pm 14.2$ \\
Gender, $\mathrm{n}(\%)$ & \\
\hline Male & $44(59.5)$ \\
\hline Female & $30(40.5)$ \\
\hline Ethnicity, $\mathrm{n}(\%)$ & $61(82.4)$ \\
\hline White & $4(5.4)$ \\
\hline Black & $3(4.1)$ \\
\hline Asian & $6(8.1)$ \\
\hline Mixed & $25.5 \pm 4.2$ \\
\hline Mean BMI kg/m ${ }^{2}$ (SD) & $18.3( \pm 10.9)$ \\
\hline Mean T1DM duration in years (SD) & $68( \pm 14.5) \mathrm{mmol} / \mathrm{mol}$ \\
\hline Mean HbA1c at assessment (SD) & $(8.4 \pm 1.3, \%)$ \\
\hline HbA1c range & $44-110 \mathrm{mmol} / \mathrm{mol}$ \\
\hline Mean insulin requirement and dose & $(6.2-12.2, \%)$ \\
\hline at assessment, (SD) & \\
\hline Insulin requirement (0.6 units per & $46.3 \pm 8.5$ \\
kg body weight) & \\
\hline Total insulin dose & $49.8 \pm 18.1$ \\
\hline Total basal insulin & $26.5 \pm 10.9$ \\
\hline Total bolus insulin & $23.8 \pm 9.3$ \\
\hline Needle length, $\mathrm{n}(\%)$ & $29(39.1)$ \\
\hline $4 \mathrm{~mm}$ & $24(32.4)$ \\
\hline $5 \mathrm{~mm}$ & $17(23)$ \\
\hline $6 \mathrm{~mm}$ & $3(4.1)$ \\
\hline $8 \mathrm{~mm}$ & $1(1.4)$ \\
\hline B2.7 mm & \\
\hline
\end{tabular}

$\mathrm{BMI}$, body mass index; HbA1c, glycated hemoglobin; T1DM, type 1 diabetes mellitus.

\section{RESULTS}

Two-hundred and twenty-six eligible patients were screened to participate in the study, of which 133 were approached to participate, 52 declined participation and 7 could not attend the ultrasound session, leaving a sample of 74 participants. The mean age of participants was $40.6( \pm 14.2)$ years, with a mean diabetes duration of $18.3( \pm 10.9)$ years. A summary of the demographic and clinical characteristics of the 74 participants is presented in table 1.

\section{Lipohypertrophy: characteristics}

Ultrasound revealed areas of increased echogenicity in all injection sites in comparison to normal surrounding tissue. There were diffuse patches of dense tissue and nodules of varying size. The lipohypertrophic nodules and diffuse areas were then graded as shown in figure 1 . 

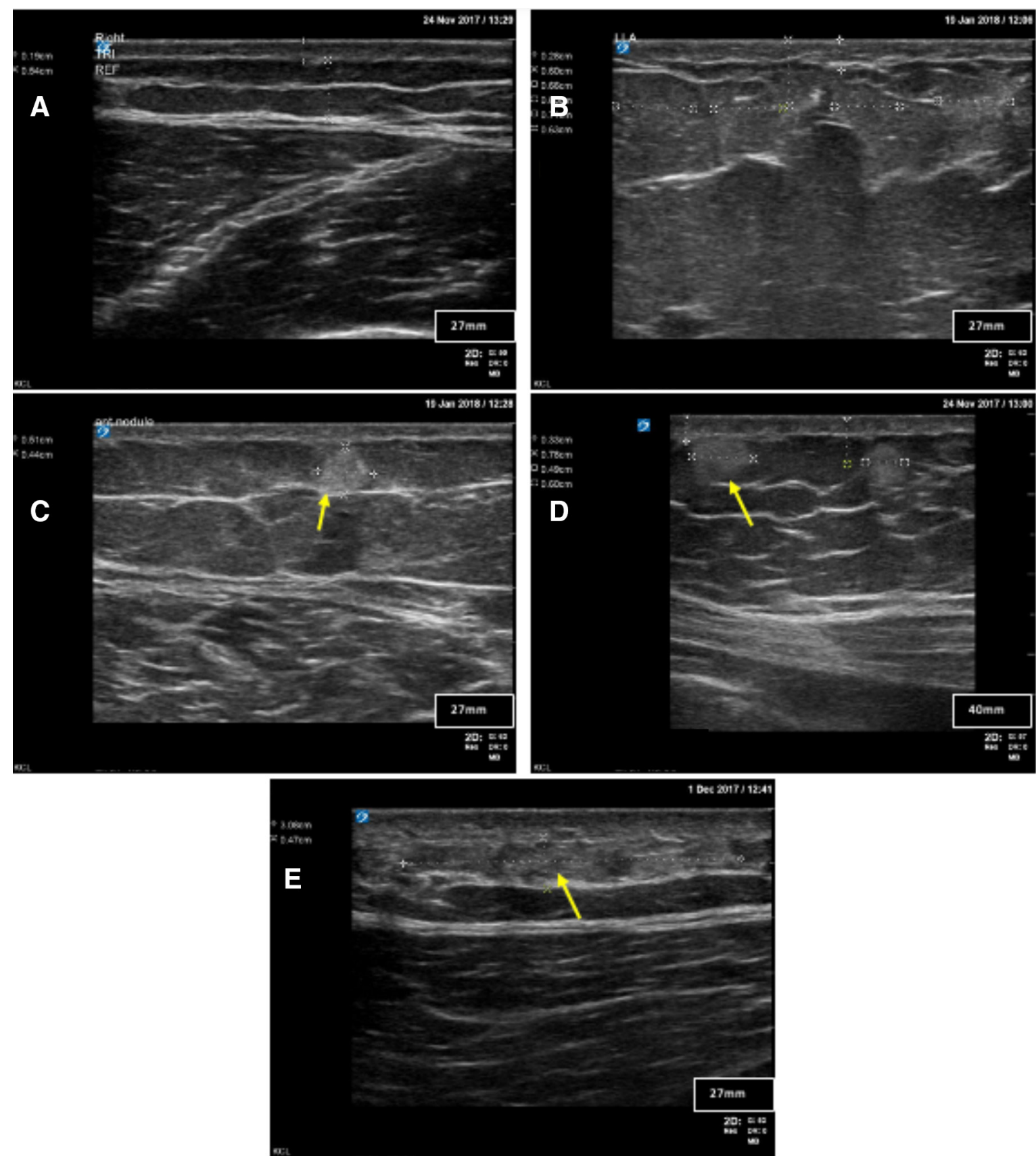

Figure 1 Characteristics of lipohypertrophy by grade.

In reviewing the ultrasound images, a number of additional observations were made in respect of lipohypertrophic nodules, and subcutaneous and dermal tissue changes. In some of the larger nodules, areas of reduced echogenicity were observed, which may indicate low blood flow, indicative of potential necrotic tissue (see figure 2, panel 1). In total, 42 areas of reduced echogenicity were identified within nodules and these were present in $30 \%(n=22)$ of participants. Delineation between the dermal layers was disrupted in all current injection sites. In some participants, the dermal disruption was marked, and the dermal layer was thicker in comparison with adjacent areas not being used as an injection site (see figure 2, panels 2 and 4). The scans showed that 52 participants $(70 \%)$ had an increased thickness of their dermal layer; this was $30 \%$ thicker in 36 of these participants $(48 \%)$ and $50 \%$ thicker in the other $16(38 \%)$ compared with their reference site. With regard to the ultrasound grading of the dermal thickness at the injection site: in the lower abdomen of the 55 participants who used this area as a site, $3.6 \%(\mathrm{n}=2)$ were grade 1 (normal), $56.4 \%(\mathrm{n}=31)$ were grade 2 and $40 \%$ $(n=22)$ were grade 3 , with a dermal layer of $\geq 3 \mathrm{~mm}$. In the 45 participants who used the thigh as a site, $6.7 \% \quad(\mathrm{n}=3)$ were grade $1,75.6 \%(\mathrm{n}=34)$ were grade 2 and $17.8 \%$ $(n=8)$ were grade 3 . In ultrasound images of two of the participants, small to medium size nodules $(1.8-6.5 \mathrm{~mm})$ were identified within the thigh, without evident diffuse 

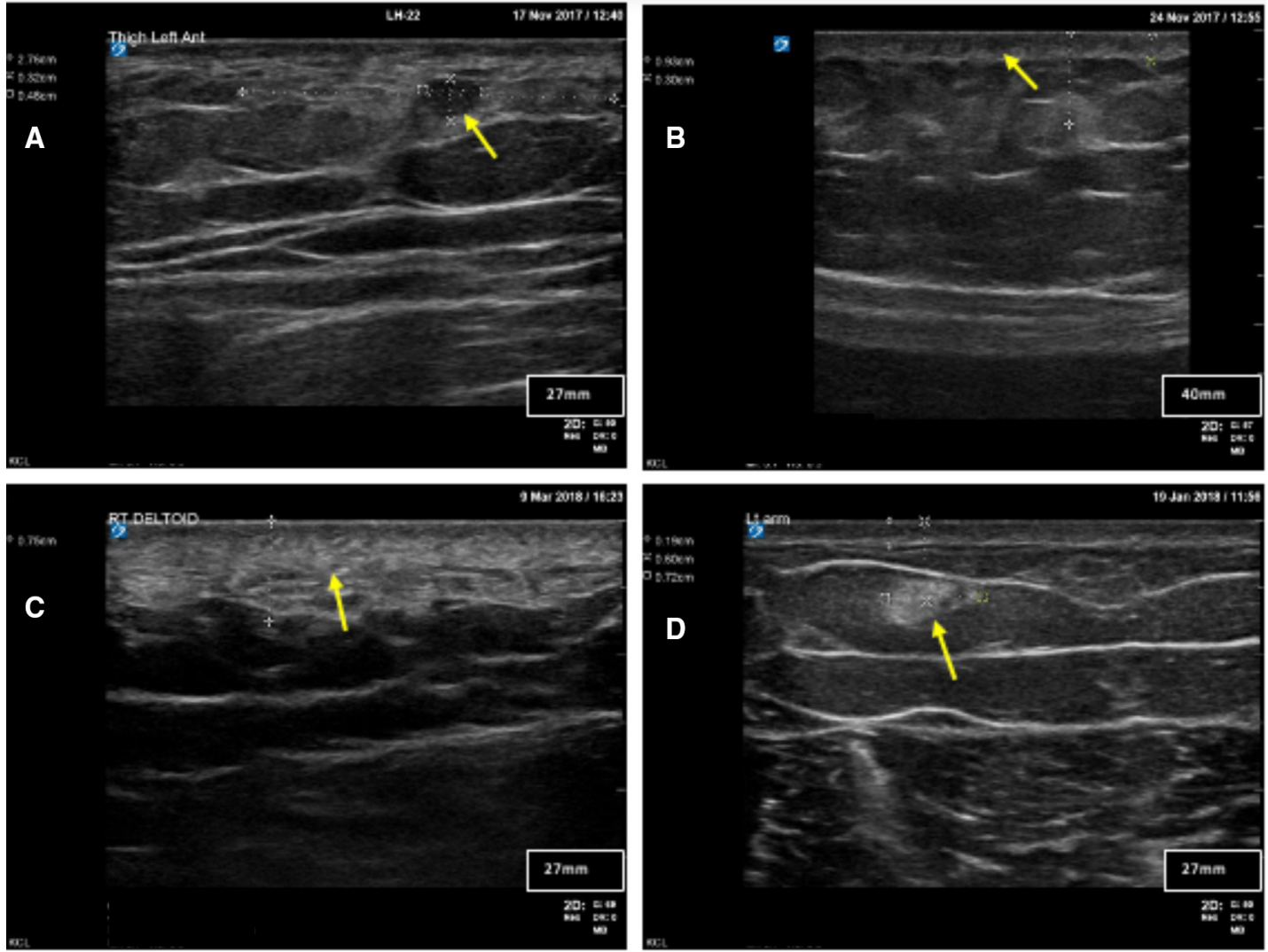

Figure 2 Additional observations.

tissue (see figure 2, panel 3). During the scanning, one of these participants confirmed that this site was rested for the last 8 years but had previously been used for 10 years. One explanation for this could be that while the diffuse tissue has dissipated due to the cessation of insulin injections in this area, nodules are more enduring. Images of these additional observations are presented in figure 2.

\section{Lipohypertrophy frequency, grade and distribution}

A total of 740 nodules were observed in the participants; the highest proportion was identified in the abdomen $(43 \%, \mathrm{n}=320)$, followed by: the thigh $(37 \%, \mathrm{n}=271)$, the gluteal region $(17 \%, \mathrm{n}=126)$ and the triceps $(3.1 \%, \mathrm{n}=23)$. In terms of the maximum nodule grade for participants, $4 \%(n=3)$ had nodules no greater than grade $1 ; 8 \%$ $(n=6)$ no greater than grade $2 ; 53 \%(n=39)$ no greater than grade 3 ; and $35 \%(n=26)$ had grade 4 nodules. The width of the lipohypertrophic nodules ranged from 1.8 $\mathrm{mm}$ to $>40 \mathrm{~mm}$ (the limit of the probe width is $40 \mathrm{~mm}$ ), with the largest nodules clustering in the thigh area. The mean nodule widths for the thigh and abdomen were $6.6 \mathrm{~mm}( \pm 2.9)$ and $6.1 \mathrm{~mm}( \pm 3.9)$, respectively, for all nodules, and the mean of the largest nodule per person was $10.6( \pm 9.3)$ and $8.7 \mathrm{~mm}( \pm 5.1)$ in the same regions. The majority of the participants had multiple nodules: $15 \%(\mathrm{n}=11)$ had $<5$ nodules; $43 \%(\mathrm{n}=32)$ 5-10 nodules; $23 \%(\mathrm{n}=17) \quad 11-15$ nodules; and $15 \%(\mathrm{n}=11)$ had $>15$ nodules. The mean (SD and range) number of nodules observed in the participants was $10.4( \pm 6.2,1-29)$, distributed as follows: abdomen 5.7 ( $\pm 3.2,0-16)$; thigh
$5.6( \pm 3.4,0-15)$; gluteal $4.3( \pm 2.9,0-14)$; and triceps 3.3 $( \pm 2.1,0-6)$. Diffuse areas were present in most injection sites $(n=304)$.

The anatomical distribution was partially mediated by left/right hand dominance; in right-handed participants $(n=64,86 \%)$, the distribution was skewed to the right in 32 participants $(50 \%)$, while the distribution was skewed to the left in 23 participants $(36 \%)$, with remainder showing an even distribution $(n=9,14 \%)$. Of the six participants who were left handed, four had more lipohypertrophy on the left side and two participants had more lipohypertrophy on the right side of their injection areas. Finally, the depth of the midpoint of the lipohypertrophy nodules was significantly correlated to needle length $(r=0.69, p<0.001)$, with the average depth of the lipohypertrophy being within $0.28 \mathrm{~mm}(\mathrm{SD} \pm 1.17 \mathrm{~mm})$ of the needle length (see figure 3 ).

\section{Conceptual model of lipohypertrophy characteristics}

From the characteristics observed in the collected images of the participants, we have developed a conceptual model to summarize these observations in relation to the grades of lipohypertrophy and the tissue changes (see figure 4).

The model illustrates the pattern of observations from single to multiple nodules and the presence of disrupted and necrotic tissue. The model also shows some of the other novel observations made in the study such as dermal disruption and the presence of areas of reduced blood flow, indicative of necrosis. The model incorporates 


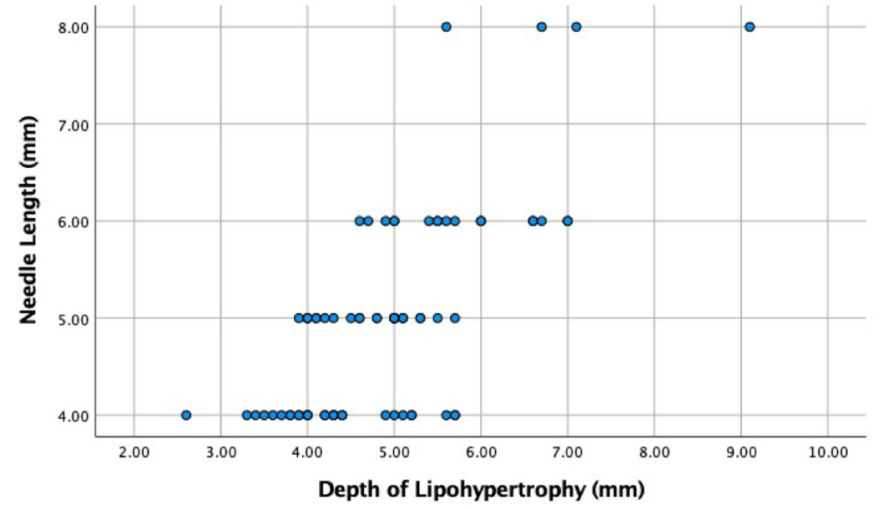

Figure 3 Corelation needle lengthdepth of lipohypertrophy.

observations from the limited number of histological studies of lipohypertrophy (discussed further), such as hypertrophy and hyperplasia of adipocytes.

\section{DISCUSSION}

While ultrasound has been used in previous studies for the detection of lipohypertrophy, this is the largest study to date to use it to characterize lipohypertrophy in adults with type 1 diabetes. This study confirms and extends the observations of previous studies, identifying that, in addition to lipohypertrophic nodules, prolonged insulin exposure is associated with more general changes in the subcutaneous and dermal tissues. We also identified evidence of potential necrosis in a third of the observed nodules. Bertuzzi $e t a l^{24}$ also reported areas of hyperechogenicity relating to tissue density and areas of heightened reflectivity similar to our observations, in a study of 20 adults with type 1 diabetes. They also reported a heterogenous profile of lipohypertrophic characteristics, with variability in the number and size of lipohypertrophic nodules alongside other subcutaneous changes, although they did not measure nodule size. A recent study by Wang et $a l,{ }^{25}$ which included ultrasound observations on the injection sites of 30 participants with type 1 diabetes, had similar observations to ours, reporting: nodular areas; tissue disruption; areas of heterogenous echogenicity; and areas of diminished vascularity. However, they did not present a detailed evaluation or classification of these observations. Similar findings of heterogenous lipohypertrophic features have been reported by Perciun ${ }^{26}$

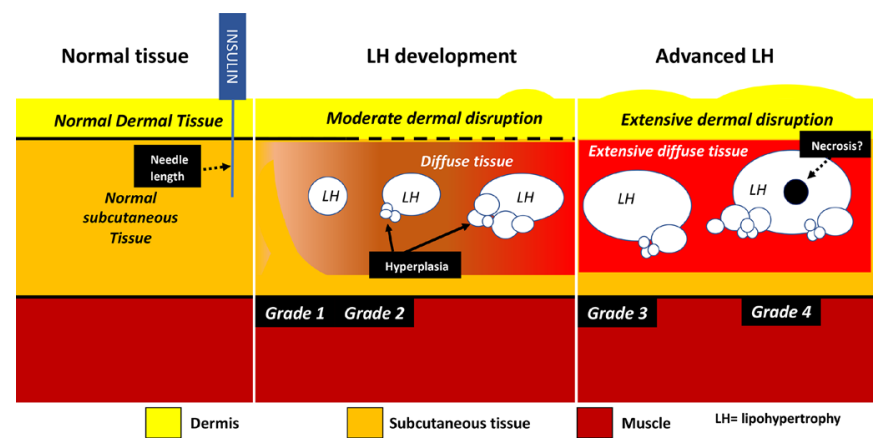

Figure 4 A conceptual model of lipohypertrophy. and Kapeluto et $a l,{ }^{27}$ although the former did not specify what type of diabetes their 40 participants had and the latter only included nine people with type 1 diabetes. A recent study from India that included 58 people with type 1 diabetes and lipohypertrophy also observed heterogenous tissue changes ${ }^{28}$ reporting increased subcutaneous thickening at injection sites with a mean of $13.0 \mathrm{~mm}( \pm 5.7)$ in the lipohypertrophic areas compared with $8.22 \mathrm{~mm}$ $( \pm 4.04)$ in the control areas. Again, they did not present a detailed characterization of the tissue changes as this was not the primary aim of the study.

Our data showed that the tissue disruption observed in insulin-exposed areas involves changes in both the subcutaneous and dermal layers, in addition to the formation of lipohypertrophic nodules. There are two potentially related mechanisms that may explain the wider dermal changes (disruption and thickening) and the diffuse tissue observed: tissue trauma and inflammation. The evidence for tissue trauma is related to the association between injection behaviours and the presence of lipohypertrophy, such as: needle reuse, poor insulin delivery technique and over injecting in limited anatomical areas. $^{12} 28-30$ Trauma and possibly localized reactions to insulin could mediate an inflammatory reaction with increased fibrosis and tissue density, which may explain the diffuse areas observed and the areas or increased reflectivity in the dermis. Inflammatory cells have been reported in lipohypertrophic tissue samples, ${ }^{31}$ with a recent histological study of lipohypertrophy in five people with diabetes showing the presence of fibrous bundles and in two cases perivascular inflammatory infiltration from lymphocytes and neutrophils. ${ }^{32}$ Rodent studies have shown that phenolic compounds found in insulin are associated with increased inflammatory stress and increased leucocyte activation. ${ }^{33}$ Insulin antibodies have also been associated with the development of lipohypertrophic tissue. ${ }^{34} 35$ It may also be that in people with higher insulin antibodies, localized insulin exposure mediates an inflammatory response causing tissue change. However, these data remain limited, and further studies to confirm the impact of inflammation on insulin exposed tissues are required.

While it is not possible to establish from ultrasonic examination that the hypoechogenic areas observed within nodules were necrotic with certainty, evidence of necrotic tissue has been reported in histological examinations of lipohypertrophic tissue. ${ }^{36}$ In addition, a recent cross-sectional study of adolescents and young adults $(\mathrm{n}=95)$ with type 1 diabetes found participants with lipohypertrophy had significantly higher levels of Tumour-Necrosis Factor alpha (TNF- $\alpha),{ }^{35}$ which is associated with necrosis. ${ }^{37}$ Further studies examining histology of lipohypertrophy-affected tissue and associated inflammatory biomarkers would be informative in confirming these observations in larger samples. An additional consideration in relation to within nodule changes was highlighted in a case study from Gentile $e t a l^{38}$ which showed areas of a colliquative nature on ultrasound, 
from which $1.5 \mathrm{~mL}$ of serous fluid was extracted indicating potential insulin pooling. Advanced imaging techniques (compression elastography, Doppler scanning, MRI and thermography) are also beginning to reveal more on the structural and vascular changes that are associated with lipohypertrophy. ${ }^{39}$ While these observations are restricted to small case reports, they generally concur with our observations showing tissue changes consistent with dermal thickening, inflammation, reduced vascular activity and potential necrosis. Future studies may consider these techniques to provide more detailed images of the structures, density and nature of lipohypertrophy.

The anatomical clustering observed in the study illustrates that lipohypertrophic tissue is largely concentrated in a few sites, predominantly the lower abdomen and outer thighs. There was also some evidence of hand dominance in clustering, as reported in previous studies. ${ }^{40}$ This would suggest that lipohypertrophic clustering is at least partially behaviorally mediated, as has also been reported in other studies. This observation emphasizes the importance of supportive education on insulin site behavior, ${ }^{41}$ although there is also evidence that any education needs regular boosting. ${ }^{42}$ There are also some emerging electronic technologies that could support/reinforce injection behaviors such as rotation. ${ }^{43}$

An additional and potentially clinically important observation in relation to the anatomical distribution of the observed nodules was that the depth of nodules, at the midpoint, correlated to the length of needle used for insulin injections. This suggests that altering needle length for periods of time could potentially increase the distribution of insulin across the available subcutaneous tissue, although the depth of an individual's subcutaneous tissue would be a limiting factor. A study of site rotation and needle length change (from $5 \mathrm{~mm}$ to $8 \mathrm{~mm}$ needles to $4 \mathrm{~mm}$ needles) in 346 people with diabetes reported a $6.3 \mathrm{mmol} / \mathrm{mol}(0.6 \%)$ $(5.5-7.2 \mathrm{mmol} / \mathrm{mol},(0.50 \%-0.66 \%), 95 \% \mathrm{CI})$ reduction in HbAlc, although this could also have been explained by the increased site rotation. ${ }^{30}$

The study also introduced a novel grading system for lipohypertrophy based on ultrasound observations, providing more granularity compared with earlier grading systems derived from visualisation or clinical measurement. ${ }^{1445}$ While we were not able to assess or validate the ultrasound-grading system against an external clinical metric such as glycemic control or glucose variability, it was possible to apply the grading consistently in assessing the lipohypertrophic areas. While our grading model shares some similarities with the ultrasound-based grading model of Perciun, ${ }^{26}$ who proposed five grades of lipohypertrophy, it is perhaps more comprehensive as it integrates the diffuse tissue with nodule width and the presence of necrotic tissue. It would now be useful to compare these two ultrasound-grading systems in terms of clinical application and to consider whether the grade indicates a risk for altered insulin action or glucose variability. If such studies were to establish the clinical benefit of the ultrasound-grading model, then a case for adopting ultrasound in routine examination of insulin injection sites could be considered. However, it is important to acknowledge that this technology would be unattainable for many low-income to middle-income countries, where cost and the supportive infrastructure required for the technology would be preclusive. Hence, manual palpation will remain an important method of clinical assessment, and indeed, it has been shown that when health professionals are well trained and follow an explicit protocol, the sensitivity gap can be minimimal, ${ }^{46}$ although in general studies show much lower sensitivity for palpation compared with ultrasound..$^{20}$

Given the complex and heterogenous tissue changes that we and others have observed in relation to insulin exposure, we might have to reconsider the terminology used to describe these changes. While lipohypertrophy is the most significant and clinically obvious element of the changes associated with excess insulin exposure, it does not fully represent what is observed. Perhaps a more accurate term would be localized insulin-exposed tissue change or damage. As a basis for this change, we developed a conceptual model of the pathological features of lipohypertrophy and the wider tissue changes we observed. While the model incorporates the grading levels, it is important to emphasize this is purely for reference as we cannot model the clinical progression of lipohypertrophy from cross-sectional observations. Indeed, the rate of developing lipohypertrophy may be quite rapid, occurring within 6 months of insulin exposure in some patients. ${ }^{46}$ In addition, nodule size may not in isolation indicate clinical severity, as some participants had multiple smaller nodules whereas others had fewer larger nodules. Therefore, what is now required is to establish the clinical implications of these lipohypertrophy classifications, in relation to glucose regulation, insulin action, risk of hypoglycemia, glycemic control and insulin requirements.

\section{Study limitations}

The findings of this study need to be considered in relation to the following limitations. The participants in this study had clinically suspected problems with lipohypertrophy. Hence, the incidence of the observed nodules and tissue changes reported cannot be generalized to the wider diabetes population, although it is likely that these observations are common as current estimates suggest that around $40 \%$ of people with type 1 diabetes have clinically identified lipohypertrophy. It is also important to emphasize that the grading system used may not be indicative of clinical severity as outlined in the discussion and that both the grading and conceptual models need further exploration and validation against metabolic outcomes. Hence, the conceptual model should be seen as a summary of what is currently known about the tissue changes associated with localized insulin exposure. In terms of future exploration, additional studies are required to: confirm the presence of inflammatory and 
necrotic tissue and to identify the clinical significance of these observations in relation to glucose regulation and insulin effect.

There were also some technical limitations to consider in how we measured lipohypertrophic nodules. We measured the width of nodules at the widest point to indicate size. The rationale for this was that we were limited to measuring in one plane rather in three dimensions (3D), as the advanced imaging techniques required for 3D assessment are currently prohibitively expensive for a study of this scale. While we could have measured hight and width and provide a measure $\mathrm{cm}^{2}$, we did not do this as the nodules were generally asymmetric and as the width measurement is in the horizontal plane, it gives a better indication of the area covered by the nodule. Finally, we did not record needle reuse, which would have been useful in interpreting the dermal thickness and subcutaneous changes observed as blunt needles can damage tissue.

\section{CONCLUSIONS}

This study has shown that insulin-related tissue changes are heterogenous in nature involving lipohypertrophy and more generalized changes within the dermal and subcutaneous tissues. We have used these observations to build a conceptual module to inform future studies and clinical understanding. We have also generated an ultrasound-grading tool for assessing tissue changes. However, it is now essential to establish the interaction between insulin mediated tissue changes and clinical outcomes to assess the point at which these changes are likely to impede glycemic control and glucose regulation.

Contributors RH: data collection and analysis, and manuscript preparation; HM: data collection (ultrasound scanning) and analysis; HAG: literature review and manuscript preparation; SH-B: senior sonographer supervised data collection and interpretation of images; MD: contributed to analysis and manuscript; RR: recruitment and data collection; JK: clinical support and manuscript preparation; AF: study design, data collection and analysis and manuscript writing- this author is the guarantor of this work.

Funding The researchers would like to acknowledge the support of the clinical diabetes research team of Guy's and St Thomas' NHS Hospital Trust. The authors would like to thank the Saudi Arabian government who sponsored a PhD fellowship for one of the researchers involved in the study. The study also received funds from an internal research allocation from King's College London - no other funding was received. The authors declare no conflicts of interest in respect of the conduct of the study and its findings.

Competing interests None declared.

Patient consent for publication Not applicable.

Ethics approval The study was ethical approved by the Health Research Authority (HRA) on behalf of the NHS in England (ref: 17/L0/1242_217187).

Provenance and peer review Not commissioned; externally peer reviewed.

Data availability statement Data are available on reasonable request. Data from this study are available by request to the corresponding author.

Supplemental material This content has been supplied by the author(s). It has not been vetted by BMJ Publishing Group Limited (BMJ) and may not have been peer-reviewed. Any opinions or recommendations discussed are solely those of the author(s) and are not endorsed by BMJ. BMJ disclaims all liability and responsibility arising from any reliance placed on the content. Where the content includes any translated material, BMJ does not warrant the accuracy and reliability of the translations (including but not limited to local regulations, clinical guidelines, terminology, drug names and drug dosages), and is not responsible for any error and/or omissions arising from translation and adaptation or otherwise.

Open access This is an open access article distributed in accordance with the Creative Commons Attribution Non Commercial (CC BY-NC 4.0) license, which permits others to distribute, remix, adapt, build upon this work non-commercially, and license their derivative works on different terms, provided the original work is properly cited, appropriate credit is given, any changes made indicated, and the use is non-commercial. See: http://creativecommons.org/licenses/by-nc/4.0/.

ORCID iDs

Janaka Karalliedde http://orcid.org/0000-0002-2617-8320

Angus Forbes http://orcid.org/0000-0003-3331-755X

\section{REFERENCES}

1 Hauner H, Stockamp B, Haastert B. Prevalence of lipohypertrophy in insulin-treated diabetic patients and predisposing factors. Exp Clin Endocrinol Diabetes 1996;104:106-10.

2 Clemmons DR. Metabolic actions of insulin-like growth factor-I in normal physiology and diabetes. Endocrinol Metab Clin North Am 2012;41:425-43.

3 Anderson JM, Rodriguez A, Chang DT. Foreign body reaction to biomaterials. Semin Immunol 2008;20:86-100.

4 Shive MS, Anderson JM. Biodegradation and biocompatibility of PLA and PLGA microspheres. Adv Drug Deliv Rev 1997;28:5-24.

5 Wang K, Zhang S, Liu C, et al. A meta-analysis and meta-regression on the prevalence of lipohypertrophy in diabetic patients on insulin therapy. Therapie 2021:617-28.

6 Famulla S, Hövelmann U, Fischer A, et al. Insulin injection into lipohypertrophic tissue: blunted and more variable insulin absorption and action and impaired postprandial glucose control. Diabetes Care 2016;39:1486-92.

7 Johansson U-B, Amsberg S, Hannerz L, et al. Impaired absorption of insulin aspart from lipohypertrophic injection sites. Diabetes Care 2005;28:2025-7.

8 Thow JC, Johnson AB, Marsden S, et al. Morphology of palpably abnormal injection sites and effects on absorption of isophane(NPH) insulin. Diabet Med 1990;7:795-9.

9 Heinemann L. Insulin absorption from lipodystrophic areas: a (neglected) source of trouble for insulin therapy? J Diabetes Sci Technol 2010:4:750-3.

10 Hovelmann U, Famulla S, Hermanski L. Insulin injection into regions with lipohypertrophy (LHT) worsens postprandial (PP) blood glucose (BG) versus injections into normal adipose tissue (nat). Diabetes Care 2016;64:A254-5.

11 Strollo F, Guarino G, Armentano V, et al. Unexplained hypoglycaemia and large glycaemic variability: skin lipohypertrophy as a predictive sign. Diabetes Res Open J 2016;2:24-32.

12 Blanco M, Hernández MT, Strauss KW, et al. Prevalence and risk factors of lipohypertrophy in insulin-injecting patients with diabetes. Diabetes Metab 2013;39:445-53.

13 Ibarra LS-D, Gallego F. Factors related to lipohypertrophy in insulintreated diabetic patients: role of educational intervention. Practical Diabetes International 1998;15:9-11.

14 Deeb A, Abdelrahman L, Tomy M, et al. Impact of insulin injection and infusion routines on lipohypertrophy and glycemic control in children and adults with diabetes. Diabetes Ther 2019;10:259-67.

15 Ji L, Sun Z, Li Q, et al. Lipohypertrophy in China: prevalence, risk factors, insulin consumption, and clinical impact. Diabetes Technol Ther 2017;19:61-7.

16 Hajheydari Z, Kashi Z, Akha O, et al. Frequency of lipodystrophy induced by recombinant human insulin. Eur Rev Med Pharmacol Sci 2011;15:1196-201.

17 FIT. Forum for injection technique (fit): the UK injection technique recommendations. 4th edn, 2016. http://www.fit4diabetes.com/files/ 4514/7946/3482/FIT_UK_Recommendations_4th_Edition.pdf

18 Frid A, Hirsch L, Gaspar R, et al. The third injection technique workshop in Athens (titan). Diabetes Metab 2010;36 Suppl 2:S19-29.

19 Seyoum B, Abdulkadir J. Systematic inspection of insulin injection sites for local complications related to incorrect injection technique. Trop Doct 1996;26:159-61.

20 Abu Ghazaleh H, Hashem R, Forbes A, et al. A systematic review of ultrasound-detected lipohypertrophy in insulin-exposed people with diabetes. Diabetes Ther 2018;9:1741-56.

21 Mulnier H, Hashem R, Duaso M. Subcutaneous tissue changes and dermal inflammation at insulin injections sites: a feasibility study using ultrasound to describe characterise and grade lipohypertrophy [Abstract]. Diabetologia 2017;60:S90 https://www. easd.org/virtualmeeting/home.html\#!resources/subcutaneous- 
tissue-changes-and-dermal-inflammation-at-insulin-injections-sitesa-feasibility-study-using-ultrasound-to-describe-characterise-andgrade-lipohypertrophy

22 Kaplan PA, Matamoros A, Anderson JC. Sonography of the musculoskeletal system. AJR AMJ Roentgeno 1990;155:237-45.

23 Gibney MA, Arce $\mathrm{CH}$, Byron KJ, et al. Skin and subcutaneous adipose layer thickness in adults with diabetes at sites used for insulin injections: implications for needle length recommendations. Curr Med Res Opin 2010;26:1519-30.

24 Bertuzzi F, Meneghini E, Bruschi E, et al. Ultrasound characterization of insulin induced lipohypertrophy in type 1 diabetes mellitus. $J$ Endocrinol Invest 2017;40:1107-13.

25 Wang W, Tong Y, Chen Y. Evaluation of ultrasound examination combined with intensive injection technique education on insulininduced lipohypertrophy (LH) management: a prospective cohort study in China. Int J Diabetes Dev Ctries 2021;16:1-7 https:// www.springermedizin.de/evaluation-of-ultrasound-examinationcombined-with-intensive-inj/18865316

26 Perciun R. Ultrasonographic aspect of subcutaneous tissue dystrophies as a result of insulin injections. Med Ultrason 2010;12:104.

27 Kapeluto JE, Paty BW, Chang SD, et al. Ultrasound detection of insulin-induced lipohypertrophy in type 1 and type 2 diabetes. Diabet Med 2018;35:1383-90.

28 Barola A, Tiwari P, Bhansali A, et al. Insulin-Related lipohypertrophy: lipogenic action or tissue trauma? Front Endocrinol 2018;9:638.

29 De Coninck C, Frid A, Gaspar R, et al. Results and analysis of the 2008-2009 insulin injection technique questionnaire survey. $J$ Diabetes 2010;2:168-79.

30 Grassi G, Scuntero P, Trepiccioni R, et al. Optimizing insulin injection technique and its effect on blood glucose control. J Clin Trans/ Endocrinol 2014;1:145-50.

31 Fujikura J, Fujimoto M, Yasue S, et al. Insulin-Induced lipohypertrophy: report of a case with histopathology. Endocr $J$ 2005;52:623-8.

32 Klimontov VV, Lazarev MM, Letyagin AJ, et al. Lipodystrophy at the insulin injection sites: current trends in epidemiology, diagnostics and prevention. Diabetes mellitus 2020;23:161-73.

33 Kesserwan S, Mulka A, Sharafieh R, et al. Advancing continuous subcutaneous insulin infusion in vivo: new insights into tissue challenges. J Biomed Mater Res A 2021;109:1065-79.

34 Raile K, Noelle V, Landgraf R, et al. Insulin antibodies are associated with lipoatrophy but also with lipohypertrophy in children and adolescents with type 1 diabetes. Exp Clin Endocrinol Diabetes 2001;109:393-6.
35 Singha A, Bhattacharjee R, Dalal BS, et al. Associations of insulininduced lipodystrophy in children, adolescents, and young adults with type 1 diabetes mellitus using recombinant human insulin: a cross-sectional study. J Pediatr Endocrinol Metab 2021;34:503-8.

36 Wallymahmed ME, Littler P, Clegg C, et al. Nodules of fibrocollagenous scar tissue induced by subcutaneous insulin injections: a cause of poor diabetic control. Postgrad Med $J$ 2004;80:732-3.

37 Ruan H, Lodish HF. Insulin resistance in adipose tissue: direct and indirect effects of tumor necrosis factor-alpha. Cytokine Growth Factor Rev 2003;14:447-55.

38 Gentile S, Strollo F, Corte TD, et al. Skin complications of insulin injections: a case presentation and a possible explanation of hypoglycaemia. Diabetes Res Clin Pract 2018;138:284-7.

39 Klimontov VV, Lazarev MM, Letyagin AJ, et al. Lipodystrophy at the insulin injection sites: current trends in epidemiology, diagnostics and prevention. Diabetes mellitus 2020;23:161-73. In Russ.

40 Gentile S, Guarino G, Corte TD, et al. Insulin-Induced skin lipohypertrophy in type 2 diabetes: a multicenter regional survey in southern Italy. Diabetes Ther 2020;11:2001-17.

41 Gentile S, Strollo F, Nefrocenter Research Study Group. Cost saving effects of a short-term educational intervention entailing lower hypoglycaemic event rates in people with type 1 diabetes and lipohypertrophy. Diabetes Res Clin Pract 2018;143:320-1.

42 Gentile S, Guarino G, Della Corte T, et al. The durability of an intensive, structured Education-Based rehabilitation protocol for best insulin injection practice: the ISTERP-2 study. Diabetes Ther 2021;12:2557-69.

43 Klarskov CK, Hamid YH, Tjalk-Bøggild R, et al. A new medical device for improved rotation of insulin injections in type 1 diabetes mellitus: a proof-of-concept study. J Diabetes Sci Technol 2021;15:1111-20.

44 Conwell LS, Pope E, Artiles AM, et al. Dermatological complications of continuous subcutaneous insulin infusion in children and adolescents. J Pediatr 2008;152:622-8.

45 Kordonouri O, Lauterborn R, Deiss D. Lipohypertrophy in young patients with type 1 diabetes. Diabetes Care 2002;25:634.

46 Gentile S, Guarino G, Giancaterini A, et al. A suitable palpation technique allows to identify skin lipohypertrophic lesions in insulintreated people with diabetes. Springerplus 2016;5:563.

47 Omar MA, El-Kafoury AA, El-Araby RI. Lipohypertrophy in children and adolescents with type 1 diabetes and the associated factors. BMC Res Notes 2011;4:1-3. 